\title{
A Simple and Practical Double Fast Excitation Circuit of Magnetic Control Reactor
}

\author{
MA Tao ${ }^{1, a}$, WANG Hai ${ }^{2, b}$, LI Chun-dong ${ }^{3, c}$, GUAN Shi-shen ${ }^{4, d}$, YANG Ping ${ }^{5, e}$ \\ ${ }^{1}$ Department of automation Wuchang University of Technology Information and Engeneering school, \\ Wuhan, HuBei 420223 , P.R. China \\ 1,3,4,5Liaoning Electric Power Development Stock Co.,LTD, Shenyang 110179,Liaoning Province \\ China \\ ${ }^{2}$ State Grid Shenyang Electric Power supply Company, Shenyang 100300, Liaoning Province China \\ a,b,c,d,e Inmt0408@163.com
}

Keywords: Magnetic control reactor; Excitation transformer; Thyristor; Double quick response.

\begin{abstract}
Efforts to improve the response speed of the magnetic control reactor, expand the scope of application of magnetic control reactor is urgently needed. This paper introduces a consists of both wave controlled rectifier circuit of double excitation circuit, not only can enhance the response speed of the magnetic control reactor, also has the circuit features of simple structure, convenient control and good economical efficiency, particularly suitable for large capacity fast excitation of magnetic control reactor.
\end{abstract}

\section{Introduction}

In recent years, the scope of application of magnetic control reactor [4] is urgently needed. However, the slow response speed of magnetic control reactor also stands out. Such the sufficiency makes the application of magnetic control reactor become limited. Efforts to improve response speed of magnetic control rector from two aspects of enhancing design of rectifier circuit and adopting digital control have already obtained positive achievements. There are many successful cases that control response speed of small and middle-sized magnetic control reactor within in 40 ms.

For high-capacity magnetic control reactor, especially for ultra-large capacity one, the difficulty of improving its response speed is larger than magnetic control reactor with small and middle-sized capacity. This is determined by the structure of magnetic control reactor and iron core's volume of magnetic control reactor.

Under the situation of a certain structure and iron core of magnetic control reactor, how to improve response speed of magnetic control reactor still is the important task of magnetic control reactor's depth development.

The basic measure of improving response speed of magnetic control reactor is to improve structure and exciting mode of exciter circuit. According to the principle of reducing transient process of magnetic control reactor and high-speed excitation principle [11-15], this paper puts forward the circuit scheme of dual excitation and applies in the magnetic control reactor of 30Mkvar and 7Mvar, etc. The response speed is no less than $30 \mathrm{~ms}$ and obtains satisfactory excitation effects.

\section{Structure and Working Process of Dual Exciter Circuit}

Dual exciter circuit is mainly constituted by excitation transformer, silicon controlled rectifier and power consumption resistance, etc., as shown in Figure 1. In the figure, B is exciting transformer. VT1 VT4 is high-power silicon controlled rectifier. $\mathrm{R}$ is power consumption resistance of exciter circuit. VD is fly-wheel diode. Ld is field winding of magnetic control reactor, namely the load of controlled rectifying circuit. 


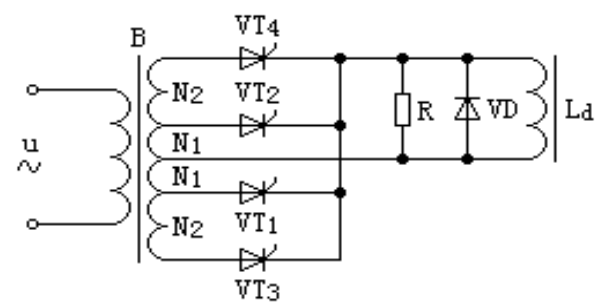

Fig.1 Fast excitation circuit topology

It can be observed from the figure that secondary winding of excitation transformer $\mathrm{B}$ is equipped with the center tap, and UN1 < UN2(generally speaking, UN2=10UN1). For the N1 winding and center tap of excitation transformer, silicon controlled rectifier VT1 and VT2 constitute low voltage import full wave controlled rectifying circuit. For the N2 winding and center tap of excitation transformer, silicon controlled rectifier VT3 and VT4 constitute low voltage import full wave controlled rectifying circuit. For winding and center tap of excitation transformer, silicon controlled rectifier constitutes high voltage import full wave controlled rectifying circuit. Two sets of rectifying circuits constitute dual exciter circuit. In order to narrate conveniently, these two sets of rectifying circuits are called as low voltage rectifying circuit and high voltage rectifying circuit.

When magnetic control reactor stays in stability, low voltage rectifying circuit can provide exciting current for field winding Ld. At this moment, silicon controlled rectifier VT1 and VT2 are triggered to be breakover, while silicon controlled rectifier VT3 and VT4 are closed.

When needing to increase exciting current, namely to reduce reactance value of magnetic control reactor, high voltage rectifying circuit turns to provide exciting current for field winding Ld. At this moment, silicon controlled rectifier VT3 and VT4 are triggered, while silicon controlled rectifier VT1 and VT2 are closed.

When needing to increase reactance value of magnetic control reactor, namely to reduce exciting current, it returns to low voltage rectifying circuit, which provides exciting current for field winding. Work transition of high voltage and low voltage rectifying circuits is controlled in line with numerical value demand of network reactive compensation by control system. Figure 2 and Figure 3 describe the working process of low voltage rectifying circuit and high voltage rectifying circuit, respectively.

Figure 2(a) is the rectifying process, when silicon controlled rectifier VT1 stays in positive half period of rectified voltage UN1. Figure 2(b) is the rectifying process, when silicon controlled rectifier VT2 stays in positive half period of rectified voltage UN1. VT1 and VT2 are triggered alternatively and output full wave direct current. It can be observed from the figure that this is the typical full wave controlled rectifying circuit, because import rectified voltage UN1 $<\mathrm{UN} 2$. Therefore, Figure 2 is called as low voltage rectifying circuit.

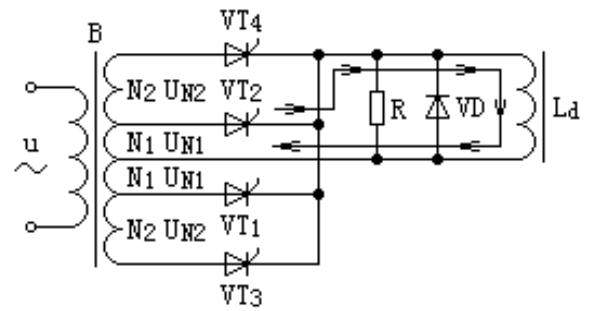

(a)

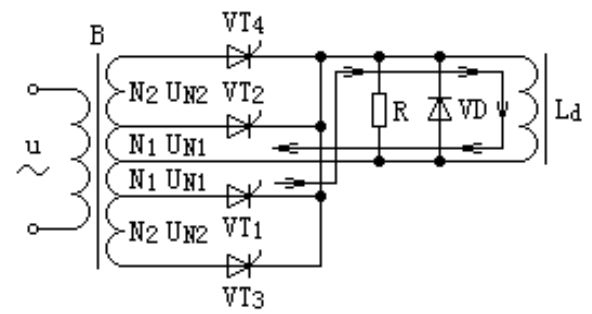

(b)

Fig. 2 The working process of the low voltage rectifying circuit 
The working principle of Figure 3 is the same with the Figure 2. Figure 3(a) is the rectifying process, when silicon controlled rectifier VT4 stays in positive half period of rectified voltage UN2. Figure3 (b) is the rectifying process, when silicon controlled rectifier VT3 stays in positive half period of rectified voltage UN2. Because of UN2 $>$ UN1, Figure (3) is called as high voltage rectifying circuit. Moreover, due to UN2>UN1, high voltage rectifying circuit can provide larger exciting current for field winding.

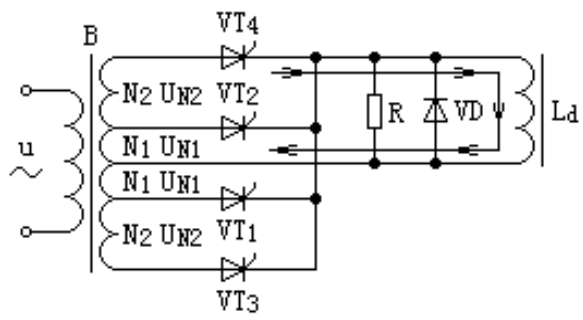

(a)

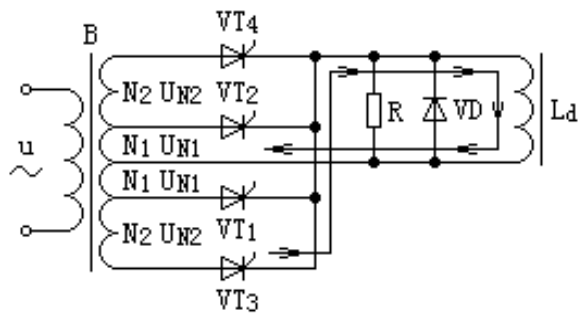

(b)

Fig.3 The working process of the high voltage rectifying circuit

It can be observed from Figure 1 that this dual exciter circuit can output high and low output voltage just by using an excitation transformer and four silicon controlled rectifiers and can output exciting current of different sizes to field winding. Moreover, because these 4 silicon controlled rectifiers have the connection characteristic of the same negative, so it just need two trigger signals, dispensing with isolation, to satisfy trigger demands of silicon controlled rectifiers. Thus, it greatly simplifies trigger circuit and improves the reliability of exciter circuit.

3. Principle Basis of High-Speed Excitation

After field winding of magnetic control reactor inputs step DC voltage, the time constant is:

$$
\tau=\frac{\mu U_{c} H_{k}^{2}}{P_{k}},
$$

In the formula, $P_{k}$ is the consumption power in DC circuit, $P_{k}=I_{k}^{2} R_{k} ; U_{c}$ is iron core's volume, $U_{c}=S_{c} L_{c} ; \mu$ is magnetic conductivity.

The formula indicates that when iron core parameter is certain, time constant of magnetic control reactor is in direct proportion to iron core's volume, while is in inversely proportional to DC circuit power consumption. It can reduce time constant and improve rapidness of magnetic control reactor by enhancing power consumption of magnetic control reactor. In the Figure 1, resistance $\mathrm{R}$ is power consumption resistance. Adjustment of resistance $\mathrm{R}$ can improve response speed of magnetic control reactor.

\section{Test Result and Industrial Application of Dual Exciter Circuit}

Figure 4 and Figure 5 are electric current step waveforms of the magnetic control reactor under the function of dual excitation. Figure 4 is the step waveform, when magnetic control reactor's electric current rises. Figure 5 is the step waveform, when magnetic control reactor's electric current falls down. It can be observed from waveforms that no matter it is the process of increasing magnetic control reactor's electric current, or the process of reducing magnetic control reactor's electric current, transient process of electric current step is less than 30ms. It indicates that dual excitation can reduce 
the response time of magnetic control reactor and improve the response speed of magnetic control reactor significantly.

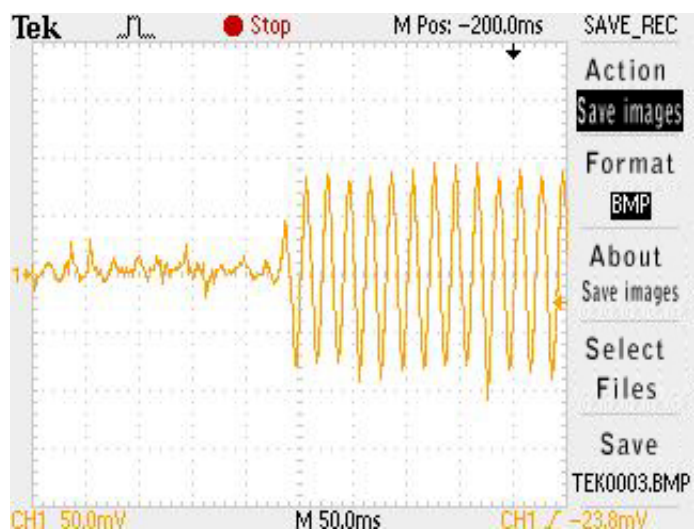

Fig.4 The rising waveform of magnetically controlled reactor current

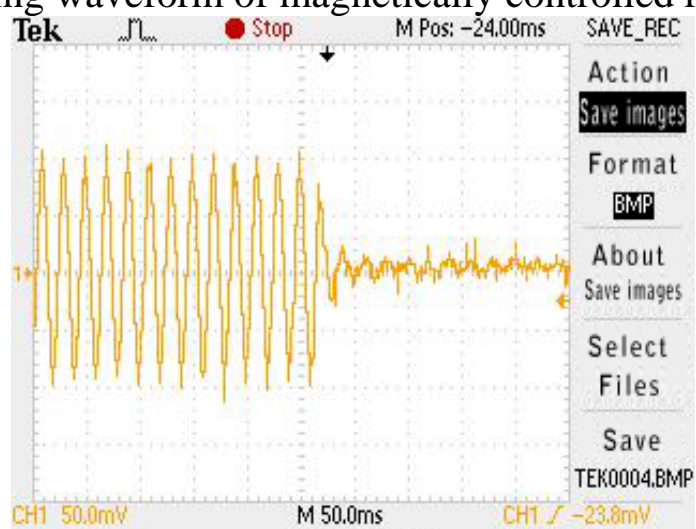

Fig. 5 The falling waveform of magnetically controlled reactor current

In 2013, dual excitation technology is applied to high-speed excitation of 30Mvar and 7Mva's magnetic control reactors, respectively. After one year of operation and checkout, dual excitation technology not only indicates excellent high-speed performance, but also shows good stability. In order to further assess high-speed excitation performance of dual excitation technology and conduct tests on wind electricity reactive compensation of magnetic control reactor.

\section{Elimination of Field Winding Induced Voltage}

From the perspective of theoretical significance, when magnetic control reactor stays in steady state, both ends of field winding shouldn't have alternating voltage, namely alternating voltage at both ends of field winding ULd $=0 \mathrm{~V}$. Because magnetic control reactor's manufacturing technique has symmetry(such as, core limb sectional area's difference and assembly technique difference of the same reactor), alternating voltage at both ends of excitation winding mostly is not 0 volt. Both ends of some excitation winding have hundreds of volts or even higher alternating voltage. When electric current of magnetic control reactor takes place step, this alternating voltage still needs to be further enlarged. The result may cause that silicon control rectifier that should have been triggered has higher alternating voltage in the cathode and can't be triggered. It also makes silicon controlled rectifier that should have be cut off continue to be triggered, because it can't be cut off, so as to break the normal working time series of controlled rectifying circuit. When it is serious, it may cause result in chain reaction of damaging silicon controlled rectifier and excitation transformer.

The effective way of eliminating alternating voltage at both ends of field winding is to use exercise strict control cover assembly technique of magnetic control reactor and reduce the voltage to the minimum value(i.e. not more than $10 \mathrm{~V}$ ). In addition, there is neutralizing transformer between direct-current output end of exciter circuit and input end of field winding(i.e. transformer B2 in the Figure 6). It is an effective action to eliminate alternating voltage at both ends of field winding. 


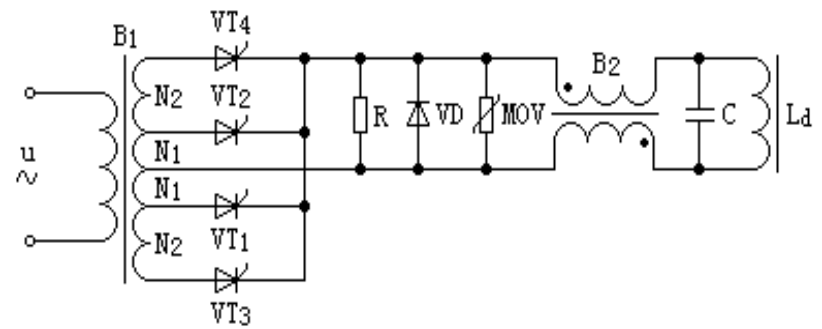

Fig.6 Using offset transformer to eliminate the induction voltage excitation wind

Lots of experiments show that application of neutralizing transformer can neutralize hundreds of voltages alternating voltage into several tiny voltages, exempting from the threat of alternating voltage at both ends of field winding on exciter circuit. Because neutralizing transformer offsets alternating voltage from field winding, it has no effect on transmittal direct current exciting current and has tiny effect on response speed of magnetic control reactor. In order to enhance offset effect of neutralizing transformer on alternating voltage, two windings of neutralizing transformer should realize tight coupling under the premise of satisfying insulation requirements.

Capacitor $\mathrm{C}$ in the Figure 6 is set for providing exchanging channel. In order to not impact excitation speed, value of capacitor $\mathrm{C}$ should range from $0.1 \mu \mathrm{F}$ to $1 \mu \mathrm{F}$. In the Figure $6, \mathrm{MOV}$ is voltage dependent resistor and is used for implementing overvoltage protection on exciter circuit.

\section{Conclusions}

Through lots of experimental measurement and operation practice checkout of multiple magnetic control reactors, the dual excitation scheme proposed by the paper has bright characteristic of high-speed excitation and shortens response time of magnetic control reactor within 30 ms. Thus, magnetic control reactor has more superiority in wind electricity reactive compensation and elevator and rolling mill, etc., which require for high-speed reactive compensation. Moreover, because exciter circuit can realize high and low voltage excitation output by only adopting four silicon controlled rectifiers. Compared with duplex control bridge rectifying circuit, which only adopts 6 silicon controlled rectifiers, it not only reduces the number of silicon controlled rectifiers and lowers manufacturing costs of exciter circuit, but also simplifies the number of trigger signal and avoids from the trouble of isolating high voltage trigger signal. It simplifies trigger circuit and control circuit and improves the reliability of exciter circuit.

This paper introduces the basic principle of dual excitation and is also suitable for magnetic control reactor of self-excitation.

\section{References}

[1] Yu Mengze, Chen Baichao, Tian Cuihua, Qiu Hao. High Voltage Static Yar Reactive Power Compensator Using Shunt Magnetically Controllable Reactor[J] • HIGH VOLTAGE ENGINEERING, 2009, 35(7): 1770-1775.

[2] Wang Baoan, Jin Lili, Luo Yaqiao, Xu Bin, Fei Yijun, Yuan Xiaodong. Dynamic reactive power compensation equipment based on magnetically controlled reactor[J]. Electric Power Automation Equipment, 2010, 30(4): 97-100.

[3] Chen Xuxuan, Tian Cuihua, Chen Baichao, Liu Yaozhong, Yuan Jiaxin. Mathematical Model for Harmonics Analysis of the Multi-Stage Saturable Magnetic-Valve Controllable Reactor[J]. TRANSACTIONS OF CHINA ELECTROTECHNICAL SOCIETY, 2011, 26(3): 57-64.

[4] Song Jiangbao, Wang Heping, Zhang Zhanyong, Zhao Guosheng - Analytical study of controllable reactor based on three-phase magnetism valve type[J]. POWER SYSTEM PROTECTION AND CONTROL, 2009, 37(23): 20-22, 40. 
[5] Zhao Shishuo, Yin Zhongdong, Liu Haipeng. Physical Design and Control Method of Fast Response Magnetically Controlled Reactors[J]. Proceedings of the CSEE, 2013, 33(15): 149-155.

[6] Liu Haipeng, Yin Zhongdong, Li Heming, Cao Songwei . Improvement of SMCR response[J]. Electric Power Automation Equipment, 2014, 34(5): 116-120.

[7] $\mathrm{Xu} \mathrm{Yi,} \mathrm{Chen} \mathrm{Jianye.} \mathrm{Numerical} \mathrm{and} \mathrm{Experimental} \mathrm{Analysis} \mathrm{of} \mathrm{Magnetically} \mathrm{Controlled}$ Saturated Reactor[J]. CONVERTER TECHNOLOGY \& ELECTRIC TRACTION, 2008, 3: 9-15.

[8] Wang Fengxiang, Zhang Tao, Bai Haoran. Multi-phase rectifying system using phase-shifting reactor[J]. JOURNAL OF SHENYANG UNIVERSITY OF TECHNOLOGY, 2008, 30(4): 361-365, 393.

[9] LI Heming, Liu Haipeng, Yin Zhongdong. Design of Control System Based on Magnetically Controlled Reactor[J]. High Voltage Engineering, 2014, 40(1): 275-281.

[10] Zheng Tao, Zhao Yanjie, Jin Ying, Zhang Zhenhuan, Cui Dawei, Liu Yang. Analysis on and Protective Schemes Against Control Winding Turn-to-turn Fault of Magnetic Controllable Shunt Reactors[J]. Automation of Electric Power Systems, 2014, 38(10): 95-99.

[11] Cai Xuansan, Liu Yuenong. Controllable saturated reactor theory,design and application [M]. Beijing: China Water Power Press, 2008: 44-52, 104-127.

[12] Chen Baichao. The new Controlled Saturated Reactor Theory and Application[M]. Wuhan: Wuhan university of hydraulic and electric engineering press, 1999: 70-107.

[13] Tian Mingxing, Li Qingfu, Wang Shuhong,The magnetic saturation type controlled reactor is equivalent physical model and mathematical model[J]. Journal of electrotechnics, 2002, 17(4): 18-21.

[14] Wei Yunbing, Li Tao, Zhang Guoliang, Li Ping, Cui Guangzhao. A control method for the magnetic-valve controllable reactor based on instantaneous reactive power theory[J]. Power System Protection and Control, 2011, 39(22): 117-121.

[15] Chen Zhenhu, Liang Jiyong,, Huang Xiangwei. Design and application of power system dynamic MCR reactive power compensation device based on[J]. POWER SYSTEM TECHNOLOGY, 2005, 29(7): 82-84. 